\title{
TU Muscae and the Early-type Overcontact Binaries
}

\author{
Dirk Terrell \\ Department of Space Studies, Southwest Research Institute, Boulder, \\ CO 80301, USA
}

\begin{abstract}
A simultaneous photometric and spectroscopic analysis of the purported early-type overcontact binary TU Muscae is presented. With a well-behaved light curve and a mass ratio far from unity, if $\mathrm{TU}$ Mus is indeed an overcontact system, it presents an interesting challenge for 3-D stellar evolution codes, like the Djehuty code, that will be applied to early-type overcontact binaries. The analysis shows TU Mus is slightly overcontact and that a detailed treatment of the reflection effect is important for modeling the light curves.
\end{abstract}

\section{Introduction}

One of the major unresolved issues in close binary star research is the structure and evolution of overcontact binaries. ${ }^{1}$ After approximately a decade of vigorous research from the late 1960's to the late 1970's, precipitated by Lucy's (1968) seminal paper on the subject, progress on understanding these stars has slowed. There are probably many reasons for this lack of progress, but the main one is that the necessary computing power to model these stars in three dimensions has only recently been developed. A full treatment of overcontact binaries will require a 3-D code capable of modeling the hydrodynamics, energy transport, and probably the magnetic fields present. Such a code, called Djehuty, is currently under development at the Lawrence Livermore National Laboratory by Peter Eggleton and his LLNL colleagues, and will be applied to modeling overcontact binaries.

Of course, the goal of such codes is to model real binaries so that we can test the results. For effective testing, we need accurate data on the properties of observed systems with a variety of different fundamental parameters, namely total mass, mass ratio, and orbital period. Modern data analysis tools like the Wilson-Devinney program (Wilson \& Devinney 1971; Wilson 1979; Wilson 1990) provide the means to extract those parameters from the observations, primarily multi-bandpass light curves and radial velocity curves.

It is natural to split the overcontact systems into those with common convective envelopes (CCE) and those with radiative ones (CRE). Almost all of the theoretical work on contact binaries has been targeted at the CCE systems, but CRE systems are not at all uncommon among early-type binaries. In order to

\footnotetext{
${ }^{1}$ See Wilson (2001) for a discussion of the name 'overcontact.'
} 
provide good tests for future theoretical models of CRE systems, the author has begun to analyze the available data for these systems as well as obtain new data. The first system studied under this program is the CRE system TU Muscae.

\section{Previous Work on Overcontact Binary Models}

Kuiper (1941) showed that the mass-radius relation for two zero-age main sequence stars

$$
\left(\frac{R_{1}}{R_{2}}\right)=\left(\frac{M_{1}}{M_{2}}\right)^{\alpha}
$$

with

$$
\alpha=0.6
$$

was inconsistent with the relation from the Roche model

$$
\left(\frac{R_{1}}{R_{2}}\right)=\left(\frac{M_{1}}{M_{2}}\right)^{0.46}
$$

except in the trivial case of equal masses. But unevolved systems with mass ratio different from 1 do exist, leading to the "Kuiper Paradox." TX Cancri has a mass ratio of about 0.5 and is a member of the Praesepe cluster several magnitudes below the turn-off point.

Lucy (1968) showed that the Kuiper Paradox could be circumvented with convective common envelopes. In such systems the adiabatic parts of the convection zones will be in contact and have equal adiabatic constants. Since the adiabatic constants are equal, they will necessarily be different from the values for single stars of the same masses. It has long been known (Schwarzschild 1958) that the radius of a star with a convective envelope is sensitive to the value of the adiabatic constant. Thus Lucy was able to demonstrate the existence of zero-age contact configurations. But despite his success in developing zero-age models that matched observed light curves very well, other predictions of the model, such as the observed period-color relation, were not so good.

In a later paper Lucy (1976) introduced the Thermal Relaxation Oscillation (TRO) theory. Since unevolved systems cannot achieve thermal equilibrium, they will oscillate about a state of marginal contact. In the TRO model, the system will oscillate between overcontact and semi-detached configurations. Robertson \& Eggleton (1977) showed that the semi-detached stage was shortlived, so that an EW light curve would be exhibited most of the time with short intervals where the system would have an EB light curve. Thus systems in the semi-detached state would be rare finds. Lucy \& Wilson (1979) presented three systems that could be interpreted as being in the broken-contact phase.

In contrast to the significant efforts to model CCE systems, very little progress has been made in modeling CRE systems. Lucy (1968) argued that Kuiper's argument was probably correct in the case of CRE systems and that unevolved overcontact systems could not exist. So the key question is whether or not they are observed. 


\section{TU Muscae}

TU Mus is a reasonably well-observed system that is a good candidate for CRE status. Various investigators (Stickland et al. 1995; Hilditch \& Bell 1987; Andersen \& Grønbech 1975) agree that the spectral type is around O8. It has a period of about 1.39 days and is about 8th magnitude in $\mathrm{V}$, making it an easy target for spectroscopy. Andersen \& Grønbech give well-sampled and high quality uvby light curves and radial velocities from optical spectra. Stickland et al. (1995) give radial velocities from IUE spectra. And there was decent coverage of the light curve by Hipparcos. Surprisingly, none of these data had been analyzed with a modern light curve synthesis code until the current study.

The analysis of the data was carried out with a soon-to-be-released version of the Wilson-Devinney (WD) program (Wilson \& Devinney 1971; Wilson 1979; Wilson 1990). Two key capabilities of this version vis a vis application to TU Mus are the ability to model filter bandpasses (rather than using a monochromatic effective wavelength) and the inclusion of Kurucz atmospheres. The new version of WD does a much better job of fitting ultraviolet light curves than previous versions.

The analysis was carried out on the Stickland et al. (1995) radial velocities, the Andersen \& Grønbech photometry, and the Hipparcos photometry. The data were fit simultaneously with weights based inversely on the scatter of the observations. Several parameters, such as the gravity darkening exponent and the bolometric albedo, were set to the theoretically expected values. Limb darkening was modeled using the logarithmic law and the coefficients from Van Hamme (1993). Time, rather than phase, was used as the independent variable, enabling the investigation of changes in the ephemeris over time. Finally, the temperature of star 1 was assumed to be $34,000 \mathrm{~K}$ based on the spectral classification. Table 1 lists the adjusted parameters.

Table 1. Adjusted parameters in light and velocity curve fits.

\begin{tabular}{cl}
\hline Symbol & Meaning \\
\hline$a$ & semimajor axis \\
$P$ & orbital period \\
$H J D_{0}$ & time of primary minimum \\
$v_{\gamma}$ & systemic velocity \\
$i$ & inclination \\
$T_{2}$ & temperature of star 2 \\
$\Omega_{1}$ & potential of common surface \\
$q$ & mass ratio $m_{2} / m_{1}$ \\
$L_{1}$ & luminosity of star 1 in a given bandpass \\
\hline \hline
\end{tabular}

One interesting result of the light curve modeling was that using a detailed treatment of the reflection effect (viz. Wilson 1990) turned out to be important. In the detailed reflection treatment, multiple iterations can be specified. A better fit to the TU Mus photometry was achieved with five iterations as opposed to two. Experiments with nine iterations showed no improvement over five. 


\section{The Parameters of TU Muscae}

The main result of the data analysis is that TU Mus is indeed an overcontact binary, although just marginally so. For the estimated mass ratio of 0.634 , the critical potential is 3.126 and the estimated potential of the surface of the common envelope is $3.118 \pm 0.004$. Table 2 shows the full list of estimated parameters.

Table 2. Estimated values for the adjusted parameters.

\begin{tabular}{cll}
\hline Parameter & Estimated Value & Standard Error \\
\hline$a$ & $16.00 \mathrm{R}_{\odot}$ & 0.02 \\
$v_{\gamma}$ & $-14.6 \mathrm{~km} \mathrm{~s}^{-1}$ & 0.3 \\
$H J D_{0}$ & 2448500.3069 & 0.0002 \\
$P$ & 1.38728638 & 0.00000008 \\
$i$ & 77.1 & 0.1 \\
$T_{2}$ & $30,200 \mathrm{~K}$ & 100 \\
$q$ & 0.634 & 0.002 \\
$\Omega_{1}$ & 3.118 & 0.004 \\
$L_{1} /\left(L_{1}+L_{2}\right)_{H i p p a r c o s}$ & 0.655 & 0.003 \\
$L_{1} /\left(L_{1}+L_{2}\right)_{u}$ & 0.667 & 0.003 \\
$L_{1} /\left(L_{1}+L_{2}\right)_{v}$ & 0.655 & 0.002 \\
$L_{1} /\left(L_{1}+L_{2}\right)_{b}$ & 0.653 & 0.002 \\
$L_{1} /\left(L_{1}+L_{2}\right)_{y}$ & 0.653 & 0.002 \\
\hline
\end{tabular}

One objective of the current study was to determine the stability of the orbital period of TU Mus by using time rather than phase as the independent variable and adjusting $H J D_{0}, P$, and $\dot{P}$. Never did attempts to adjust $\dot{P}$ result in statistically significant non-zero values. Fig. 1 shows the fits to the $u$ and $y$ data of Andersen \& Grønbech from the early 1970's and Fig. 2 shows the fit to the Hipparcos data from the early 1990's. Both sets of data were phased using the ephemeris parameters from Table 2, illustrating the stability of the ephemeris over approximately twenty years. $B V$ data currently being obtained by R. H. Nelson (private communication) show no change in the ephemeris. So, unlike many overcontact binaries, TU Mus has a very stable light curve.

The fit to the data has consistent runs of residuals, especially near the maximum at phase 0.25 and the observations do show a slight asymmetry in the maxima, a common, if not well understood, feature of overcontact binaries. The asymmetry in the $u$ data is slightly larger than in the $y$ data.

The masses and radii of the components of TU Mus are under some dispute. Andersen \& Grønbech gave masses of $M_{1}=23.5 \mathrm{M}_{\odot}$ and $M_{2}=15.8 \mathrm{M}_{\odot}$ as well as radii of $R_{1}=8.0 \mathrm{R}_{\odot}$ and $R_{2}=6.6 \mathrm{R}_{\odot}$. Stickland et al. gave masses of $M_{1}=17.2 \mathrm{M}_{\odot}$ and $M_{2}=10.8 \mathrm{M}_{\odot}$. (They studied only the radial velocities and thus gave no estimate of the component radii.) Results of the current study are $M_{1}=17.5 \mathrm{M}_{\odot}, M_{2}=11.1 \mathrm{M}_{\odot}, R_{1}=6.8 \mathrm{R}_{\odot}$ and $R_{2}=5.5 \mathrm{R}_{\odot}$. Since the Stickland et al. velocities were used in this study, it is not too surprising that the resulting masses are in good agreement with theirs. This rather large difference 

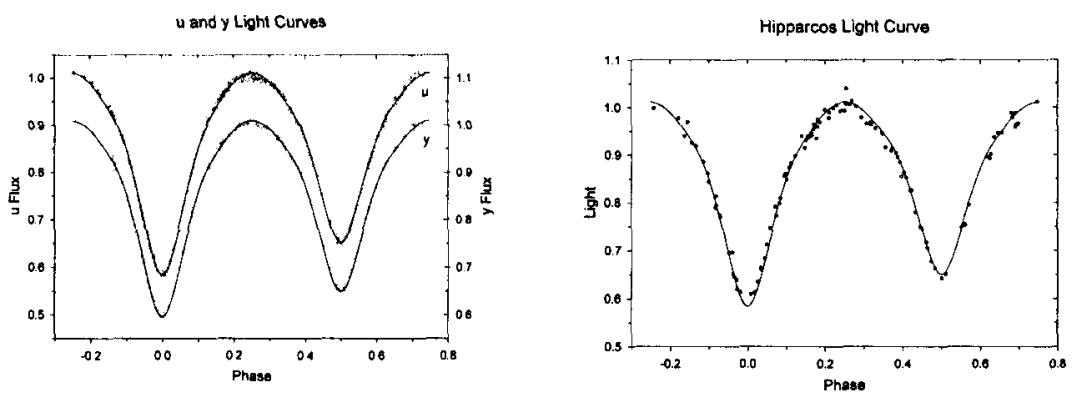

Figure 1. Fits to the Andersen \& Grønbech (1975) $u$ and $y$ data (left) and the Hipparcos data (right).

in masses obtained with optical spectra and those obtained from ultraviolet data is disturbing and has been noted by Stickland and co-workers in other systems. Efforts are currently underway to obtain high resolution $(R=50,000)$ optical spectra of TU Mus and other early-type overcontact systems.

\section{Conclusions}

TU Mus is an overcontact system, although barely. Perhaps the most remarkable feature of the system is the stability of the light curve over time. TU Mus is a well-behaved system and thus a good target of 3-D modeling efforts.

\section{References}

Andersen, J., \& Grønbech, B. A\&A, 45, 107

Hilditch, R. W., \& Bell, S. A. 1987, MNRAS, 229, 529

Kuiper, G. 1941, ApJ, 93, 133

Lucy, L. B. 1968, ApJ, 151, 1123

Lucy, L. B. 1976, ApJ, 205, 208

Lucy, L. B., \& Wilson, R. E. 1979, ApJ, 231, 502

Robertson, J. A., \& Eggleton. P. P. 1977, MNRAS, 179, 359

Schwarzschild, M. 1958, Structure and Evolution of the Stars (Princeton: Princeton University Press)

Stickland, D. J., Lloyd, C., Koch, R. H., \& Pachoulakis, I. 1995, The Observatory, 115,317

Van Hamme, W. 1993, AJ, 106, 2096

Wilson, R. E. 1979, ApJ, 234, 1054

Wilson, R. E. 1990, ApJ, 356, 613

Wilson, R. E. 2001, IBVS, 5076

Wilson, R. E., \& Devinney, E. J. 1971, ApJ, 166, 605 